\title{
Strong, weak and metastable liquids
}

\author{
H.N.W. Lekkerkerker* \\ Van't Hoff laboratory for Physical and Colloid Chemistry, Debve Research Institute, Utrecht University. \\ Padualaan 8, $3584 \mathrm{CH}$ Utrecht, The Netherlands
}

\begin{abstract}
A discussion is given on the effect of the ratio (range of attractive interaction)/(range of repulsive interaction) on the phase behaviour of colloidal systems, simple fluids and protein solutions.
\end{abstract}

This paper is dedicated to Ben Widom on the occasion of his 70 th birthday

\section{Introduction}

Ben Widom has made seminal contributions to our understanding of the nature of the liquid state. He clearly recognized the relative importance of the attractive and repulsive components of intermolecular forces in determining the properties of liquids near their critical points and near their triple points [1]. These notions lie at the very heart of our present understanding of the liquid state.

The equilibrium properties of colloidal suspensions may be treated in the same way as in simple liquids by considering the colloidal particles as "supramolecules" [2]. Onsager [3] showed that in applying statistical mechanics to such mesoscopic particles one must use the potential of the average forces which act between these particles. However, colloids show a range of fascinating new phenomena not encountered in atomic systems related to the fact that the interactions between colloidal particles can be adjusted or "tailored". This lets us investigate experimentally how the behaviour of an assembly of particles depends on their interaction. This paper is devoted to a few remarks on the effect of the ratio

$$
\frac{\text { range of attractive interaction }}{\text { range of repulsive interaction }} \text {. }
$$

\footnotetext{
*E-mail: lekkerkerker@chem.ruu.nl.
} 


\section{Phase behaviour of colloid-polymer mixtures}

As was first realized by Asakura and Oosawa [4,5] and subsequently elaborated by Vrij [6] the addition of non-adsorbing polymer to a dispersion of colloidal particles leads to an attractive interaction. This effective interaction is based on the exclusion (depletion) of added polymer molecules from the region between two colloidal particles when their surface separation becomes smaller than the diameter of the added polymer molecules. The resulting imbalance in osmotic pressure gives rise to an effective attraction (depletion interaction) between the colloidal particles (Fig. 1).

Representing the colloids as hard spheres and the polymer coils as non-additive hard spheres [7] (meaning that they do not interact with themselves but only with the colloidal particles) the potential of mean force corresponding to the depletion interaction is given by $[4,6]$

$$
W= \begin{cases}\infty, & r<\sigma_{\mathrm{c}}, \\ -\Pi_{\mathrm{p}} \cdot V_{\text {overlap }}, & \sigma_{\mathrm{c}}<r<\sigma_{\mathrm{c}}+\sigma_{\mathrm{p}}, \\ 0, & r>\sigma_{\mathrm{c}}+\sigma_{\mathrm{p}} .\end{cases}
$$

Here $r$ is the distance between the centres of the colloidal particles, $\sigma_{\mathrm{c}}$ and $\sigma_{\mathrm{p}}$ are the diameters of the colloidal particle and the polymer coil, $\Pi_{\mathrm{r}}$ is the osmotic pressure exerted by the polymers and $V_{\text {overlap }}$ is the overlap volume of the depletion zones. Noic ilıat the range of the depletion attraction depends only on the polymer size $\sigma_{\mathrm{p}}$ whereas the depth depends on the osmotic pressure $\Pi_{\mathrm{p}}$ and through $V_{\text {overlap }}$ on the colloid and polymer size $\sigma_{\mathrm{c}}$ and $\sigma_{\mathrm{p}}$ (Fig. 2).

For the thermodynamic treatment of the phase separations that occur in colloid-polymer mixtures it is convenient to consider the chemical potential $\mu_{\mathrm{p}}$ of the polymer molecules as an independent thermodynamic variable [8,9]. For this purpose, we consider an osmotic equilibrium system consisting of three compartments separated by two semi-permeable membranes, with pure solvent in I, polymer solution in II and a colloid + polymer dispersion in III (Fig. 3).

In this osmotic equilibrium system the solvent, denoted by the subscript o, has a chemical potential $\mu_{\mathrm{o}}$ throughout. The osmotic pressure $\Pi_{\mathrm{p}}$ of the polymer solution

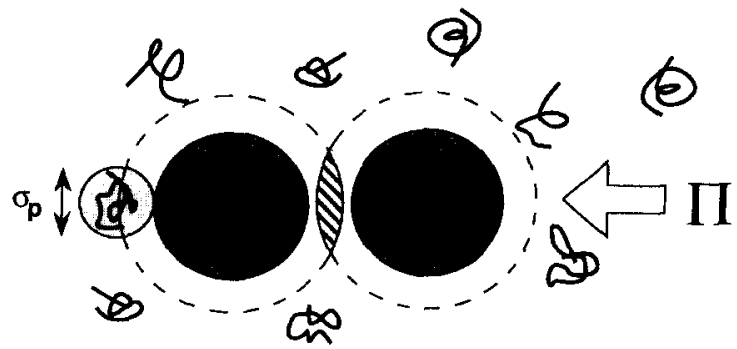

Fig. 1. The origin of the depletion potential. 


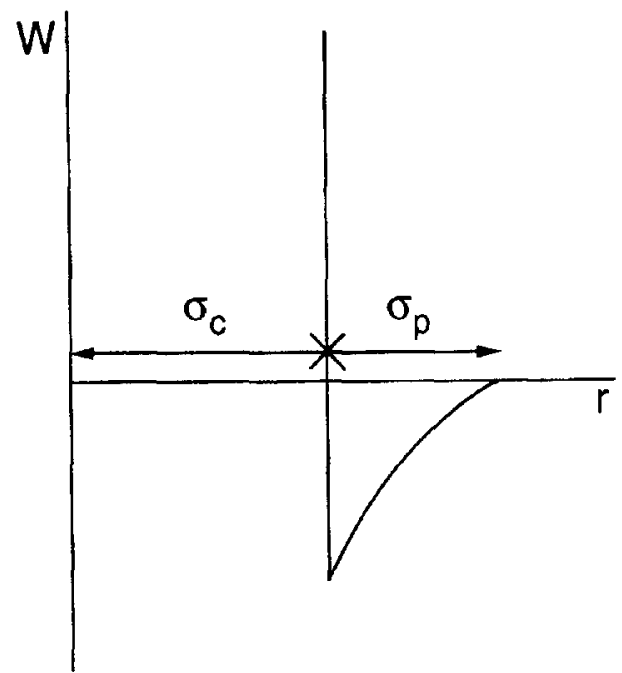

Fig. 2. The depletion potential for colloid + non-adsorbing polymer mixtures.

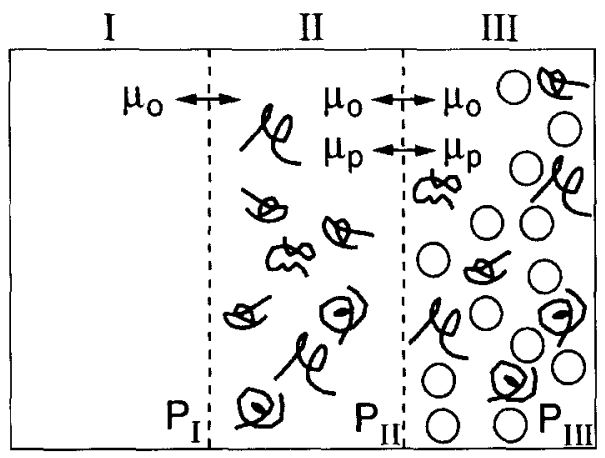

Fig. 3. Osmotic equilibrium system.

is the pressure difference between II and I

$$
\Pi_{\mathrm{p}}=P_{11}-P_{1}
$$

and the osmotic pressure $\Pi$ of the colloid-polymer mixture is the pressure difference between III and I

$$
\Pi=P_{111}-P_{1}
$$

Taking into account that the polymers are modelled as hard spheres the osmotic pressure of the polymer solution is related to the polymer activity $z_{\mathrm{p}}=\mathrm{e}^{\mu_{\mathrm{p}} / k T}$ as

$$
\Pi_{\mathrm{p}}=z_{\mathrm{p}} k T \text {. }
$$


The appropriate thermodynamic potential to describe the colloid + polymer suspension in the osmotic equilibrium system is the semi-grand potential, which is grand canonical for the solvent and polymer molecules and canonical for the colloid particles and from which the solvent contribution is subtracted [10],

$$
\begin{aligned}
\Delta \Omega\left(N_{\mathrm{c}}, V, T, \mu_{\mathrm{o}}, \mu_{\mathrm{p}}\right) & =\Omega(\text { dispersion })-\Omega(\text { solvent }) \\
& =\Omega\left(N_{\mathrm{c}}, V, T, \mu_{\mathrm{o}}, \mu_{\mathrm{p}}\right)+P_{1} V .
\end{aligned}
$$

Using the thermodynamic relation

$$
\frac{\partial \Delta \Omega}{\partial z_{\mathrm{p}}}=-\frac{k T N_{\mathrm{p}}}{z_{\mathrm{p}}}=-k T\left\langle V_{\mathrm{free}}\right\rangle,
$$

where $\left\langle V_{\text {free }}\right\rangle$ is the average free volume for a polymer coil in the system of colloidal hard spheres, and approximating it by its value in the unperturbed system of colloidal hard spheres $\left\langle V_{\text {free }}\right\rangle_{0}$ we obtain

$$
\Delta \Omega\left(N, V, T, \mu_{\mathrm{o}}, \mu_{\mathrm{p}}\right)=\Delta \Omega_{\mathrm{c}}\left(N_{\mathrm{c}}, V, T, \mu_{\mathrm{o}}\right)-\Pi_{\mathrm{p}}\left\langle V_{\text {free }}\right\rangle_{0} .
$$

In practice, $\Delta \Omega_{\mathrm{c}}\left(N_{\mathrm{c}}, V, T, \mu_{\mathrm{o}}\right)$ plays the same role for colloidal dispersions as the Helmholtz energy $A(N, V, T)$ for atomic spheres [10]. For the present case where we have modelled the colloidal particles as hard spheres we can therefore use for $\Delta \Omega_{\mathrm{c}}$ the appropriate hard-sphere expressions. The average free volume $\left\langle V_{\text {free }}\right\rangle_{0}$ for a polymer coil in the pure system of colloidal hard spheres depends only on the colloid volume fraction $\phi_{\mathrm{c}}=\left(N_{\mathrm{c}} / V\right)(\pi / 6) \sigma_{\mathrm{c}}^{3}$ and the size ratio $q=\sigma_{\mathrm{p}} / \sigma_{\mathrm{c}}$ (Fig. 4). It can be calculated conveniently from scaled particle theory [8].

The phase diagram is obtained by equating the chemical potential of the colloidal particles $\mu_{\mathrm{c}}=\partial \Delta \Omega / \partial N_{\mathrm{c}}$ and the osmotic pressure $\Pi=-\partial \Delta \Omega / \partial V$ for the coexisting phases. The activity of the polymer $z_{\mathfrak{p}}$ can be considered as a control parameter. Increasing $z_{p}$ leads to an increase of $\Pi_{p}$ and hence an increase of the strength of the attraction. Therefore, an increase in $z_{p}$ has roughly speaking the same effect as a decrease in $T$ in an atomic fluid.

The polymer-colloid size ratio $q=\sigma_{p} / \sigma_{\mathfrak{c}}$ which determines the range of the depletion attraction relative to the hard core of the colloidal particles has a remarkable effect on the phase diagram as was first observed by Gast et al. [11]. For sufficiently long-range of the depletion attraction $(q \geqslant 0.3)$ we find depending on the value of $z_{\mathrm{p}}$ the colloidal equivalents of a gas, liquid and solid phase. This is essentially equivalent to a Van der Waals fluid with the role of a decreasing temperature $T$ replaced by an increasing polymer activity $z_{\mathrm{p}}$. However, for $q \leqslant 0.3$ the gas-liquid transition is always metastable compared to the solid branch (see Fig. 5).

To compare the calculated phase diagram with experiments it is convenient to use as variable the number density of the polymer molecules in the system $\rho_{\mathrm{p}}=N_{\mathrm{p}} / V=z_{\mathrm{p}}\left(\left\langle V_{\mathrm{free}}\right\rangle_{\mathrm{o}}\right) / V$ rather than the activity $z_{\mathrm{p}}$. 


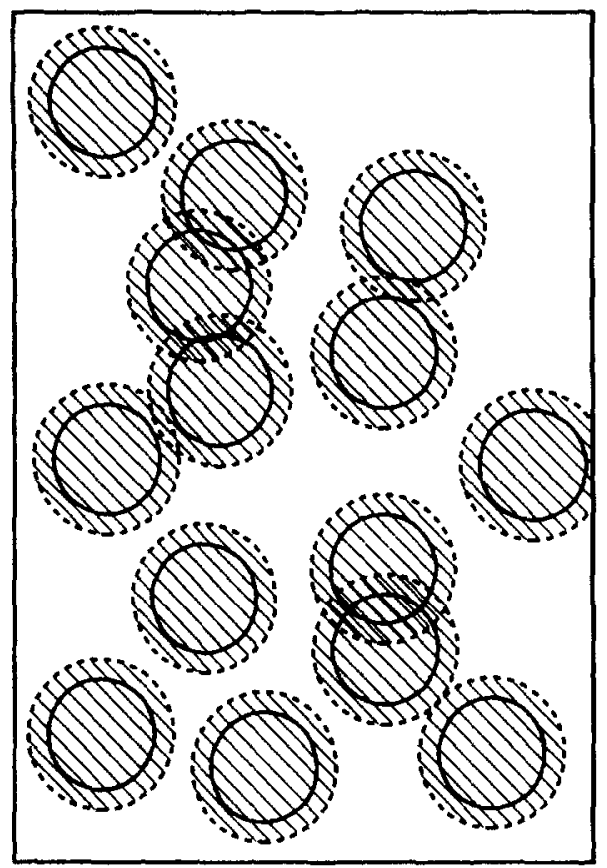

Fig. 4. Illustration of the free volume. The unshaded volume is $\left\langle V_{\text {free }}\right\rangle$.

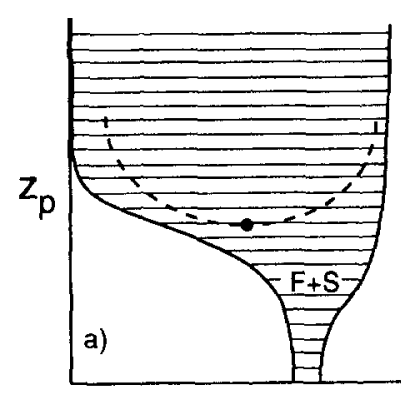

$\rho_{c}$

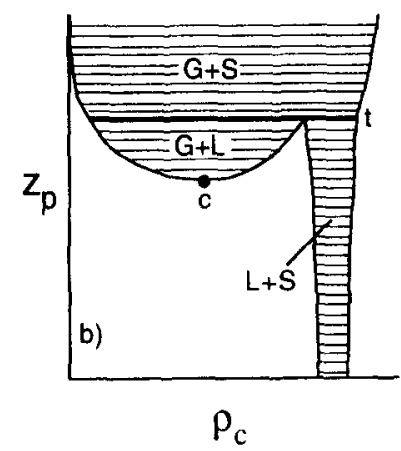

Fig. 5. Schematic phase diagram of colloid + polymer mixtures in the polymer activity $z_{\mathrm{p}}$-colloid concentration $\rho_{\mathrm{c}}$ representation: (a) $\sigma_{\mathrm{p}} / \sigma_{\mathrm{c}}<0.3$; (b) $\sigma_{\mathrm{p}} / \sigma_{\mathrm{c}}>0.3$. The dotted line represents the metastable gas-liquid coexistence. The triple line and critical point are denoted by $t$ and $c$. The letters $F, G, L$ and $S$ represent the fluid, gas, liquid and solid phase.

The resulting $\rho_{\mathrm{p}}-\rho_{\mathrm{c}}$ phase diagrams for $q<0.3$ and $q>0.3$ are given in Fig. 6.

Note that for $q>0.3$ the triple line in the $z_{\mathrm{p}}-\rho_{\mathrm{c}}$ representation transforms in a three-phase triangle bordered by three two-phase regions in the $\rho_{\mathrm{p}}-\rho_{\mathrm{c}}$ representation. This phase scenario has indeed been observed experimentally [12-14].

The metastable gas-liquid binodal which appears in colloid-polymer systems with $q \leqslant 0.3$ appears to have important consequences for the phase-transition kinetics as was clearly recognized recently by Poon et al. [15]. In fact exactly 100 years ago 

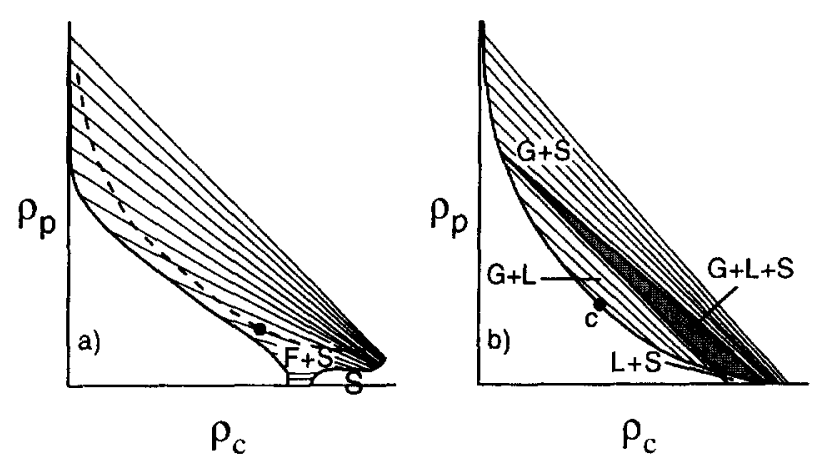

Fig. 6. Schematic phase diagram of colloid + polymer mixtures in the polymer concentration, $\rho_{\mathrm{p}}$ colloid concentration $\rho_{\mathrm{c}}$ representation: (a) $\sigma_{\mathrm{p}} / \sigma_{\mathrm{c}}<0.3$; (b) $\sigma_{\mathrm{p}} / \sigma_{\mathrm{c}}>0.3$. The symbols have the same meaning as in Fig. 5.

Ostwald [16] observed that crystallization from a solution often occurs in such a way that thermodynamically metastable phases appear first. Extending the Ostwald rule to colloid-polymer systems with $q<0.3$ one expects that for colloid and polymer concentrations inside the metastable gas-liquid binodal the gas-liquid transition would occur before the transition to the stable conjugate fluid and solid phases. However, from experiments carried out several years ago in the Van't Hoff Laboratory by Carla Smits $[17,18]$ it appears (with hindsight) that the system after having undergone the gas-liquid transition appears incapable of crystal nucleation, because it refuses to crystallize (even after a period of months). Precisely, the opposite appears to be the case in the rather narrow region between the stable fluid-solid and metastable gas-liquid transition. There crystallization proceeds much faster than in a system without added polymer. One might imagine that the critical fluctuations associated with the metastable gas-liquid critical point may lead to an increase in the rate of crystal nucleation. Recent computer simulations of Ten Wolde and Frenkel [19] provide the first evidence for this scenario.

\section{Phase diagrams of "simple" fluids with extreme pair potentials}

The question that follows naturally from the results obtained for colloid + polymer mixtures whether there are "simple" atomic or molecular substances that have no stable liquid phase. Hagen et al. [20] obtained results which suggest that $C_{60}$ might be such a substance. The phase diagram of $\mathrm{C}_{60}$ was calculated using computer simulations in which the $\mathrm{C}_{60}$ molecules are represented by spheres interacting via Lennard-Jones potentials summed over all 60 carbon atoms. The resulting intermolecular pair potential is of much shorter range than the 12-6 Lennard-Jones potential which is supposed to be a good approximation for the pair potential of the noble gases. The computer simulation results for the phase diagram of $\mathrm{C}_{60}$ show that the sublimation line passes above the gas-liquid line, which is therefore metastable. 


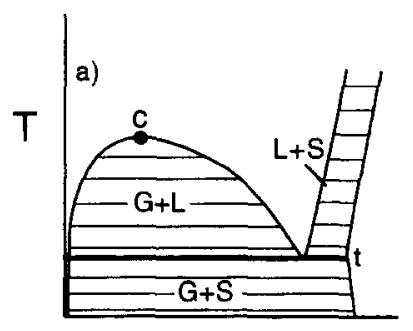

$\rho$

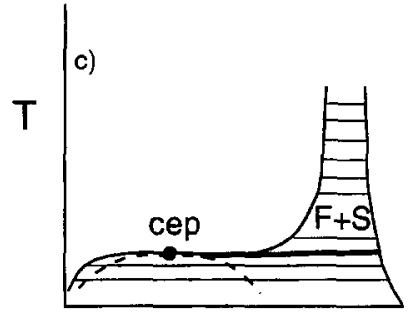

$\rho$

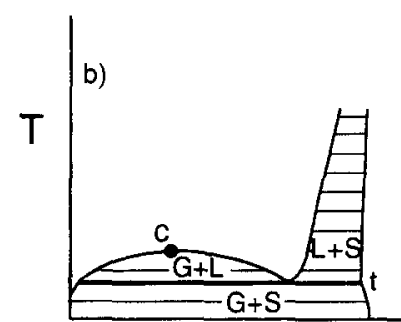

$\rho$

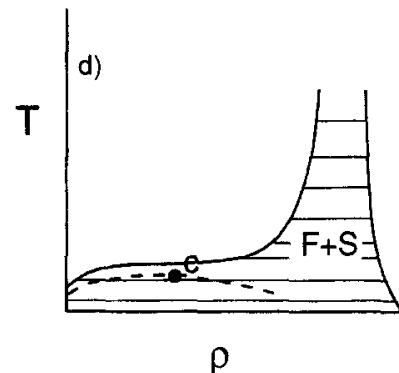

Fig. 7. Phase diagrams of a simple fluid in the temperature $T$ density $\rho$ representation. The range of attraction decreases from (a) to (d). The symbols have the same meaning as in Fig. 5 . The critical end point is denoted by cep.

Subsequently, Tejero et al. [21,22] used a simple analytical theory to calculate the phase diagram of simple fluids interacting through potentials of the double-Yukawa (DY) form with a variable range of attraction. The range of the attraction relative to the range of the repulsion is characterized by $\delta=\left(r_{1}-r_{0}\right) / r_{0}$ where $r_{1}$ is the value of $r$ for which the potential has decreased to $1 \%$ of its value at the minimum $r_{0}$. Decreasing the value of $\delta$ the progression of phase behaviour represented in Fig. 7 is obtained. For $\delta=1.34$ the ratio of the critical temperature and triple temperature is 1.8 (which is also the experimental value for argon) and the density of the liquid at the triple point is about three times the critical density. We propose to call this liquid a strong liquid (Fig. 7a). Upon decreasing $\delta$ the critical temperature $T_{\mathrm{c}}$ decreases (rapidly) and for $\delta \cong 0.5$ approaches $T_{\text {t }}$ and at the same time the density of the liquid at the triple point approaches the critical density. We propose to call this liquid a weak liquid (Fig. 7b). Finally for $\delta \leqslant 0.4$, the DY produces a phase diagram without a stable liquid phase but with a metastable gas-liquid coexistence (Fig. 7d). At the crossing from the weak liquid to the metastable liquid we have a situation where $T_{\mathrm{c}}=T_{\mathrm{t}}$ and hence the critical point turns into a critical end point (Fig. 7c). Similar results were obtained with computer simulations on $2 n-n$ Lennard-Jones potentials

$$
V(r)=4 \varepsilon\left[\left(\frac{\sigma}{r}\right)^{2 n}-\left(\frac{\sigma}{r}\right)^{n}\right]
$$


with $n$ ranging from 6-12 [23]. A remarkable feature we observed was that whereas $\left(k T_{\mathrm{c}}\right) / \varepsilon$ drops precipitously from 1.3 to 0.56 upon increasing $n$ from 6 to 12 , the value of the second virial coefficient $B_{2}$ evaluated at the critical temperature $T_{\mathrm{c}}$ is remarkably constant, changing only from $-6.2\left((\pi / 6) \sigma^{3}\right)$ for $n=6$ to $-6.4\left((\pi / 6) \sigma^{3}\right)$ for $n=12$. The significance of this fact is that, in principle, it is possible to predict from a dilute gas property, the second virial coefficient, whether the system is close to the critical temperature. The significance of this fact will be discussed in the next section.

In order to get some feeling for the (almost) constant value of $B_{2}$ at the critical temperature, regardless of the range of the attractive interaction, let us consider this quantity for two models that may be considered to represent two extremes for the range of attraction i.e. the Van der Waals model, which is valid for long (infinite)range attractions [24] and the Baxter adhesive hard-sphere model [25] where the range of the attraction goes to zero. (For a clearly argued warning about the dangers of the adhesive hard-sphere model see Stell [26].)

Using the Van der Waals expression for the pressure

$$
P=P_{\mathrm{hs}}-a \rho^{2}
$$

with the scaled particle expression for the hard-sphere pressure [27]

$$
P_{\mathrm{hs}}=\rho k T \frac{1+\phi+\phi^{2}}{(1-\phi)^{3}},
$$

one obtains for the critical point $\left(k T_{\mathrm{c}}\right) /\left(a /\left(\pi / 6 \sigma^{3}\right)\right)=0.094$ and $\phi_{\mathrm{c}}=\rho_{\mathrm{c}}(\pi / 6) \sigma^{3}=0.13$. This leads to the following result for the second virial coefficient at the critical temperature:

$$
\begin{aligned}
B_{2}\left(T_{c}\right) & =\frac{\pi}{6} \sigma^{3}\left(4-\frac{a /\left(\pi / 6 \sigma^{3}\right)}{k T_{c}}\right) \\
& =-6.66\left(\frac{\pi}{6} \sigma^{3}\right) .
\end{aligned}
$$

The Baxter adhesive hard-sphere potential is defined by the limiting case of a pair potential $V(r)$ with a hard core and square-well tail for which the well width $\delta$ goes to zero and the well depth $\varepsilon$ goes to infinity in such a way that the contribution to the second virial coefficient remains finite but not zero. This limiting case can be realized by setting

$$
\frac{V(r)}{k_{\mathrm{B}} T}=\lim _{\delta \rightarrow 0} \begin{cases}+\infty, & r<\sigma, \\ \ln \left[\frac{12 \tau \delta}{\sigma+\delta}\right], & \sigma<r<\sigma+\delta, \\ 0, & r>\sigma+\delta .\end{cases}
$$


The second virial coefficient is then

$$
\begin{aligned}
B_{2} & =2 \pi \int_{0}^{\infty}\left[1-\exp \left(-\frac{V}{k_{\mathrm{B}} T}\right)\right] r^{2} \mathrm{~d} r \\
& =\frac{\pi}{6} \sigma^{3}\left[4-\frac{1}{\tau}\right] .
\end{aligned}
$$

The critical parameters for the Baxter adhesive hard-sphere model in the Percus-Yevick approximation are using the compressibility equation [25]

$$
\phi_{\mathrm{c}}=0.121 \text { and } \tau_{\mathrm{c}}=0.098
$$

and using the energy equation [28]

$$
\phi_{\mathrm{c}}=0.320 \text { and } \tau_{\mathrm{c}}=0.12 \text {. }
$$

Using the above values for $\tau_{\mathrm{c}}$ in the expression for $B_{2}$ we obtain

$$
B_{2}\left(\tau_{c}\right)=-6.20\left(\frac{\pi}{6} \sigma^{3}\right) \text { (compressibility equation) }
$$

and

$$
B_{2}\left(\tau_{c}\right)=-4.33\left(\frac{\pi}{6} \sigma^{3}\right) \text { (energy equation). }
$$

We see that the values for the second virial coefficient at the critical temperature $T_{\mathrm{c}}$ (or critical adhesion parameter $\tau_{\mathrm{c}}$ ) for the Van der Waals fluid and Baxter adhesive hard-sphere model are fairly close to the values for $2 n-n$ Lennard-Jones potentials.

This leads me to think that for any "simple" potential (i.e. consisting of a steep repulsive part at short distance and an attractive part at larger, even albeit small, range) the second virial coefficient at the critical temperature, on a stable or metastable gas-liquid coexistence line, has a value in the range of $-4\left((\pi / 6) \sigma^{3}\right)$ to $-7\left((\pi / 6) \sigma^{3}\right)$. In the next section we will explore some of the consequences of this notion.

\section{Phase behaviour of solutions of globular proteins}

Guided by the results we obtained for $C_{60}$ [20], which has a diameter of about $1 \mathrm{~nm}$, it seems reasonable to assume that for globular molecules with sizes greater than $1 \mathrm{~nm}$ the gas-liquid transition is metastable with respect to the fluid-solid transition. Whereas apart from the fullerenes we do not know of any "simple" globular molecules with sizes greater than $1 \mathrm{~nm}$, globular proteins belong to this category.

Solutions of globular proteins such as lysozyme and calf lens $\gamma$-crystalline proteins show both liquid-liquid phase separation in dilute ("gas") and concentrated ("liquid") 
protein solution as well as solid-liquid phase separation [29-34]. In these systems the coexistence of a protein crystal phase with a protein liquid solution is indeed thermodynamically stable relative to the liquid-liquid coexistence, indicating that we are dealing here with systems where the range of attraction is smaller than say 0.25 times the range of the repulsion [35-37].

This kind of phase behaviour is expected to hold for solutions of globular proteins in general, and has important consequences for their crystallization behaviour. George and Wilson [38] presented data for a number of protein solutions which suggest that crystallization is favoured when solution conditions are such that osmotic second virial coefficient $B_{2}$ lies in a fairly narrow range. From their data we conclude that the osmotic virial coefficients in this range have values of about $-5\left((\pi / 6) \sigma^{3}\right)$ to $-8\left((\pi / 6) \sigma^{3}\right)$. This suggests that successful crystallization occurs around the top of the metastable liquid-liquid phase separation line. This is in agreement with observation in colloid-polymer mixtures $[13,15,17,18,39]$.

\section{Concluding remark}

I hope that the above examples make it clear that the effect of the ratio range of attractive interaction range of repulsive interaction

which was first studied in connection with colloid + polymer suspensions raises significant issues of theoretical and experimental interest both for the equilibriumphase behaviour as well as phase-separation kinetics in a variety of systems ranging from bucky balls to solutions of globular proteins.

\section{Acknowledgements}

I wish to thank Marc Baus, Daan Frenkel, Wilson Poon and Peter Pusey for illuminating discussions on the role of the range of the attraction on the phase behaviour of simple fluids, colloidal suspensions and protein solutions.

\section{References}

[1] B. Widom, Science 157 (1967) 375.

[2] A. Vrij, E.A. Nieuwenhuis, H.M. Fijnaut, W.G.M. Agterof, Faraday Disc 65 (1978) 101.

[3] L. Onsager, Chem. Rev. 13 (1933) 73.

[4] S. Asakura, F. Oosawa, J. Chem. Phys. 22 (1954) 1255.

[5] S. Asakura, F. Oosawa, J. Pol. Sci. 33 (1958) 183.

[6] A. Vrij, Pure Appl. Chem. 48 (1976) 471.

[7] B. Widom, J.S. Rowlinson, J. Chem. Phys. 52 (1970) 1670.

[8] H.N.W. Lekkerkerker, Colloid. Surf. 51 (1990) 419. 
[9] H.N.W. Lekkerkerker, W.C.K. Poon, P.N. Pusey, A. Stroobants, P. Warren. Europhys. Lett. 20 (1992) 559.

[10] L. Onsager, Ann. N.Y. Acad. Sci. 51 (1949) 627.

[11] A.P. Gast. C.K. Hall, W.B. Russel, J. Colloid Interface Sci. 96 (1983) 251.

[12] P.N. Pusey, W.C.K. Poon, S.M. Ilett, P. Bartlett. J. Phys.: Condens. Matter 6 (1994) A29.

[13] S.M. Ilett. A. Orrock, W.C.K. Poon, P.N. Pusey, Phys. Rev. E 51 (1995) 1344.

[14] F. Leal Calderon. J. Bibette, J. Biais, Europhys. Lett. 23 (1993) 653.

[15] W.C.K. Poon, A.D. Pirie, P.N. Pusey, Faraday Disc. 101 (1995) 65.

[16] W. Ostwald, Z. Phys. Chem. 22 (1897) 289.

[17] C. Smits, J.S. Van Dujineveldt, J.K.G. Dhont, H.N.W. Lekkerkerker, W. Briels, Phase Transit. 21 (1990) 157.

[18] C. Smits, B. Van der Most, J.K.G. Dhont, H.N.W. Lekkerkerker. Adv. Colloid Interface Sci. 42 (1992) 33.

[19] P.R. Ten Wolde. D. Frenkel, preprint.

[20] M.H.J. Hagen. E.J. Meijer, G.C.A.M. Mooij. D. Frenkel. H.N.W. Lekkerkerker, Nature 365 (1993) 425 .

[21] C.F. Tejero, A. Daanoun, H.N.W. Lekkerkerker, M. Baus. Phys. Rev. Lett. 73 (1994) 752.

[22] C.F. Tejero, A. Daanoun, H.N.W. Lekkerkerker, M. Baus. Phys. Rev. E. 51 (1995) 558

[23] G.A. Vliegenthart. H.N.W. Lekkerkerker. unpublished results.

[24] M. Kac, G.E. Uhlenbeck, P.C. Hemmer, J. Math. Phys. 4 (1963) 216.

[25] R.J. Baxter, J. Chem. Phys. 49 (1968) 2770.

[26] G. Stell. J. Stat. Phys. 63 (1991) 1203.

[27] H. Reiss, H.L. Frisch, J.L. Lebowitz, J. Chem. Phys. 31 (1959) 369.

[28] R.O. Watts, D. Henderson, R.J. Baxter, Adv. Chem. Phys. 21 (1971) 421.

[29] C. Ishimoto, T. Tanaka, Phys. Rev. Lett. 39 (1977) 474.

[30] V.G. Taratuta, A. Holschbach, G.T. Thurston, D. Blankschtein, G.B. Benedek, J. Phys. Chem. 94 (1990) 2140.

[31] M.L. Broide, T.M. Tominic, M.D. Saxowsky, Phys. Rev. E 53 (1996) 6325.

[32] J.A. Thomson. P. Schurtenberger, G.M. Thurston. G.B. Benedek. Proc. Natl Acad. Sci. USA 84 (1987) 7079

[33] M.L. Broide. C.R. Berland, J. Pande. O.O. Ogun. G.B. Benedek. Proc. Natl. Acad. Sci. USA 88 (1991) 5660.

[34] C.R. Berland. G.M. Thurston, M. Kondo, M.L. Broide, J. Pande. O.O. Ogun, G. B. Benedek. Proc. Natl. Acad. Sci. USA 89 (1992) 1214.

[35] D. Rosenbaum. P.C. Zamora. C.F. Zukoski, Phys. Rev. Letl. $76(1996) 150$

[36] N. Asheric, A. Lomakin, G.B. Benedek, Phys. Rev. Lett. 77 (1996) 4832

[37] W.C.K. Poon, Phys. Rev. E 55 (1997) 3762.

[38] A. George, W.W. Wilson, Acta Cryst. D 50 (1994) 361.

[39] W.C.K. Poon, J.S. Selfe, M.B. Robertson, S.M. Ilett, A.D. Pirie, P.N. Pusey, J. Phys. (France) II $3(1993) 1075$. 